Asian J. Med. Biol. Res. 2017, 3 (2), 145-151; doi: 10.3329/ajmbr.v3i2.33561

\author{
Asian Journal of \\ Medical and Biological Research \\ ISSN 2411-4472 (Print) 2412-5571 (Online) \\ www.ebupress.com/journal/ajmbr
}

Review

\title{
The role of iron and zinc in cognitive development of children
}

\author{
Md. Abdullah Al Mamun ${ }^{1}$ and Ruhina Binta A Ghani ${ }^{2}$ \\ ${ }^{1}$ Department of Food Technology and Nutrition Science, Noakhali Science and Technology University, \\ Sonapur-3814, Noakhali, Bangladesh \\ ${ }^{2}$ Health Nutrition and Population program, BRAC ${ }^{2}$, BRAC Centre, 75 Mohakhali, Dhaka-1212, Bangladesh
}

*Corresponding author: Md. Abdullah Al Mamun, Department of Food Technology and Nutrition Science, Noakhali Science and Technology University, Sonapur-3814, Noakhali, Bangladesh. E-mail: mamun.ftns@nstu.edu.bd

Received: 07 June 2017/Accepted: 22 June 2017/ Published: 29 June 2017

\begin{abstract}
The effects of iron and zinc on cognitive, motor and behavioral development are on children is scientifically accepted and concentrates on the more recent work, some previous work and areas of controversy. There are well established associations with poor development and iron and zinc deficiency in food source but the deficiencies usually occur in disadvantaged circumstances and establishing causal relationships is not easy. The health and well being of children depend upon the interaction between their genetic potential and exogenous factors like adequacy of nutrition, safety of the environment, social interaction and other childhood activity. Both proteins-energy malnutrition and micronutrient deficiencies increase the risk of death from common diseases such as acute gastroenteritis, pneumonia and measles. Iron deficiency anemia, for example, is estimated to affect almost $25 \%$ of the world's population resulting in high economic cost by adding to the burden on health care services, affecting learning in school. Dietary practices frequently seen in children from both developed and developing countries, leading to frequent consumption of nutrient poor foods, may also put them at risk of micronutrient deficiencies.
\end{abstract}

Keywords: cognitive development; iron; zinc; child health and development; food sources

\section{Introduction}

UNICEF (1998) defined micronutrients as nutrients that are only needed by the body in minute amounts, which play leading roles in the production of enzymes, hormones and other substances, helping to regulate growth activity, cognitive development and the functioning of the immune and reproductive systems. There are well established associations with poor development and iron and zinc deficiency but the deficiencies usually occur in disadvantaged circumstances and establishing causal relationships is difficult (Grantham et al., 1995). Micronutrients of known public health importance include the following: zinc, iodine, iron, selenium, copper, vitamins $\mathrm{A}, \mathrm{E}, \mathrm{C}, \mathrm{D}, \mathrm{B}_{2}, \mathrm{~B}_{6}$ and folate. Deficiency of these micronutrients has a significant impact on children health and cognitive development as well as on the economic development of communities and nations. The full genetic potential of the child for physical growth and mental development may become compromised due to sub clinical deficiencies of micronutrients, which are commonly referred to as "hidden hunger". Children with these sub clinical deficiencies of micronutrients are more vulnerable to develop frequent and more severe common day-to-day infections thus triggering a vicious cycle of under nutrition and recurrent infections (Singh, 2004). According to Caballero, almost two thirds of the deaths of children around the world are directly or indirectly associated with nutritional deficiencies (Caballero, 2002).

According to Singh, vitamins and trace minerals are required for production of various enzymes, hormones, and biochemical mediators for regulation of biological processes. He further stated that micronutrients are required for energy production, synthesis of RNA and DNA and for providing protection against reactive oxygen-free 
radicals. Micronutrients are also required for promotion of physical growth, sexual maturation and neuromotor development. They are recognized to boost both cell-mediated and humoral immune deficiencies of the body. Micronutrients play significant roles in the health of children. This paper therefore reviews the roles of the various micronutrients specifically iron and zinc in children as found in literature.

\section{Cognitive development}

Cognition is a complex construct and consists of a field of thought processes by which an individual registers, encodes, selects, maintains, transforms, stores and retrieves information (Shinjini and Sunita, 2001). This would extend to include visual and somato-sensory perception, thinking, memory, and learning. Memory helps to learn, retain and reproduce information. Another important aspect is attention; an integrated process by which the individual, from the time of infancy focuses on information that is essential for its growth and development (Ruff and Rauthbart, 1996). It includes a readiness to respond and an intact capacity to focus on one thing resisting other distracting stimuli. In the first year of life, the infant learns to construct mental structures, which is dependent on perceptions and body movements. It further develops its attention skills by exploring and concentrating on the novel aspects of its environment and extends it to focus on testing and organizing the information through the pre-school years Activity and motor skills form an integral part of developing the attention system by increasing the responsiveness to the environment. A lethargic child with delayed motor activity would have fewer opportunities to explore and concentrate on specific objects or events. Activity would be directly related to motor activities at that particular age. Cognitive development continues as a period of concrete operations through school years as skills like thought, memory and language develop. During this period multiple stimuli are appreciated simultaneously and attention abilities increase with greater learning and memory. Full adult intelligence and hypothetical thinking evolve during adolescence. Assessment of cognitive development would include evaluation of perception, thought, attention, memory, language and activity (Shinjini and Sunita, 2001).

\section{Nutritional needs for mental development during late pregnancy and the first two years of life}

During the last three months of gestation and early infancy, the brain is a site of high activity, where cells divide and differentiate into neurons and accessory cells. During this period of growth, proteins, lipids and energy supply is needed. Micronutrients, which serve as cofactors for many metabolic and signaling processes, are essential as well. These nutrients are normally brought prenatally by placental transfer to the fetus, and postnatally by breastfeeding (Piaget, 1997).

\subsection{Iron}

It is to keep in mind that iron is always prioritized to red blood cells over any other organ; including the brain (Denis and Denis, 2005): iron deficiency can be pathologic even before iron deficiency anemia (IDA). In other words, iron homeostasis mechanisms are made in a way which first, ensures production of red blood cells. Particularly, iron deficiency induces irreversible effects on the brain of children if occurring during pregnancy or the first year of life. One of the most important features of iron is its role in energy utilization in the respiratory chain of mitochondria (Lozoff, 2000). Studies in the rat model by De Ungria and colleagues demonstrated that iron deficiency was associated with a decreased energy metabolism in the hippocampus, a region involved in cognition and memory. This might explain the observations that iron deficiency in human neonates are associated with electrophysiological evidence of reduced recognition memory at birth (De Ungria et al., 2000). Second, iron is essential for myelin production because it is a co-factor for lipid biosynthesis. As a consequence, IDA has been shown to be associated with myelin defects and slower transmission trough the auditory and visual systems (Georgieff and Innis, 2005). This phenomenon is not reversible and persists despite treatment for IDA with iron supplementation. Finally, iron plays a role in the metabolism of monoamines, which constitute a group of neurotransmitters and neuromodulators, particularly dopamine (Algarín et al., 2003). In several studies, iron deficiencies and moderate anemia occurring during infancy were shown to affect cognitive performance at school. These studies were controlled for socio-economical background of children (UN Children Fund, 2011). In addition, iron supplementation is not always able to revert the low cognitive abilities of those children: mental development seems to occur in a "window -period" during life, inside which damages can be irreversible.

\subsection{Zinc}

Zinc is found in all human tissues but it is found at high concentrations in the brain. It is essential for the activity of a large number of metalloenzymes: the cellular functions are as wide as RNA and DNA synthesis, cellular 
growth, differentiation and metabolism. Early development -when cell activity is the highest- may be particularly sensitive to zinc deficiency. In some studies, it has been associated with reduced neuronal growth and brain volume. In addition, zinc deficiency has been shown to compromise activity, cognitive development and attention (UN Children Fund, 2011; Lozoff et al., 2000]. In some studies, zinc levels have also been correlated with reading ability and academic performances. Finally, zinc supplementation studies have shown an effect on reasoning abilities (UN Children Fund, 2011). Zinc is known to be essential for the normal growth and development of the fetus. A deficiency of this nutrient in pregnant animals has been shown to result in malformations and abnormal development and functioning of the central nervous system of the offspring (Frederickson, 1989). So malnutrition in pregnant women may affect child cognition through its effects on fetal development (Maureen, 2003). Zinc is of particular concern during pregnancy because of its potential effect on fetal brain development (Shinjini and Sunita, 2001) and birth outcomes. Fluid cognitive deficits and impaired working memory have been associated with the hippocampus (Tsunenobu Tamura et al, 2003) that has a role in spatial memory and has been suggested to be sensitive to zinc deficiency (Stoecker, 2009).

\section{Role of iron}

Iron is one of the trace minerals that play a vital role in the body. The whole body contains about $4 \mathrm{~g}$ of iron. Three-fourth of this is found in association with the protein, hemoglobin. In foods, iron occurs in two forms ferrous and ferric but the absorption form of iron is only in the ferrous state. The function of iron in the body cannot be overlooked as it is involved in the synthesis of other compounds in the body. Iron is important in reactions involving energy release in the body (oxidation and reduction reactions). It is a component of oxygen carrying compounds e.g. hemoglobin, myoglobin. Iron deficiency anemia is the most common micronutrient problem in the world as it affects more than 2 billion people globally (Black, 2003). Iron deficiency among children is common, especially in less developed countries and affects psychomotor development (Black, 2003). Infants and young children are the most adversely affected by iron deficiency because they are growing and developing at such a fast rate (Onyezili et al., 1993). If iron deficiency is not corrected, it leads to anemia, which is the most common nutritional disorder in the world. The association of iron deficiency anemia with lower mental and motor developmental test scores in early childhood is well described and has recently been reviewed (Sally and Cornelius, 2001). Draper further stated that iron deficiency anemia is a major problem affecting more than 2 billion people - more than one-third of the entire world population (Onyezili et al., 1993). The World Health Organization (WHO) also estimated that $51 \%$ of children under 4 years old in developing countries are anemic as a result of iron deficiency. According to Draper iron deficiency anemia is more common in situations of:

1. Social disadvantage (e.g. poverty, poor housing and overcrowding, and low levels of parental education),

2. Psychological disadvantage (e.g. lack of stimulation), and

3. Biological disadvantage (e.g. low birth weight, high infection rates, and other nutritional deficiencies) (Onyezili et al., 1993).

The main nutritional causes of iron deficiency anemia are diets that provide too little iron, poor absorption of most dietary iron, and the presence of other dietary factors that inhibit iron absorption. Although an inadequate iron diet is by far the major cause of anemia, it also can occur as a result of parasitic infections, inherited disorders, and deficiencies of other nutrients. Parasitic infections include malaria and helminthes (notably, hookworm). Malaria causes the destruction of red blood cells and hookworms cause blood loss: Inherited disorders that can leave an individual vulnerable to anemia include sickle cell anemia. A much less common cause for anemia results from deficiencies of other nutrients, such as folate (a B-complex vitamin) (Onyezili et al., 1993). Draper also observed that iron deficiency anemia is associated with impaired development of mental and physical coordination skills and impaired school achievement in older children (Draper, 1996). It lowers resistance to disease and weakens a child's learning ability and physical stamina. It also slows mental and motor development and reduces work performance (UNICEF, 2001).

There is evidence that iron deficiency anemia alters the emotional state of infants so that they are more withdrawn, cautious and hesitant, and maintain closer contact with their mothers. These types of behavior could hinder an infant's ability to interact with and learn from his or her environment thereby contributing to poor intellectual development. In school achievement two studies had robust designs and adequate sample sizes and examined school achievement, which has obvious policy implications (Pollitt, 1993). Children showed a clear improvement with treatment Iron deficiency anemia can be prevented in children by food fortification such as the addition of iron to food over and above that naturally found in food. Naturally iron-containing foods should not be neglected. Mothers should be encouraged to feed their infants and young children meat and foods rich in vitamin $\mathrm{C}$, such as fruits, and to avoid foods and drinks that inhibit iron absorption, such as tea, during and 2 
hours before and after mealtime. Traditional food practices such as germination and/or fermentation can also improve the availability of iron in the diet. Intake of iron supplement can as well help in reducing the prevalence of iron deficiency anemia. Iron supplements should be initiated as close to 6 months of age as possible, and supplements should be given for 6 months. Supplementation of longer duration, to 15 months of age is likely to be more beneficial (Onyezili et al., 1993).A number of public health activities can lower the risk of iron deficiency anemia. For example, in areas where malaria is endemic, the use of bed nets should be promoted.

\section{Role of zinc}

Zinc is widely recognized as an essential micronutrient with a catalytic role in over 100 specific metabolic enzymes in human metabolism (Pollitt, 1993). It is one of the most ubiquitous of all trace elements involved in human metabolism and plays multiple roles in the perpetuation of genetic materials including transcription of DNA, translation of RNA, and ultimately in cellular division (Eugene and Robert, 2005). Zinc is a trace mineral that is involved with RNA and DNA synthesis and is critical to cellular growth, differentiation and metabolism (Ozgur et al., 2004). Zinc deficiency can interfere with multiple organ systems particularly when it occurs during a time of rapid growth and development, such as infancy, when nutritional demands are high. Interest in zinc was stimulated when zinc supplements given to short children and failure to thrive infants in the U.S. city of Denver improved growth (Jill et al., 2001). Collective Ability reported that zinc is found virtually in every tissue in the body and is particularly important for the correct functioning of the immune system, growth and development and the antioxidant system (Maureen, 1998). The major source of zinc intake is through diet and when the supply of dietary zinc is insufficient, biochemical abnormalities and clinical signs may develop (Allen, 2001). The following are the major functions of zinc:

a) Zinc plays significant roles in the metabolism of proteins, carbohydrates, and lipids. It is incorporated into the active sites of many metalloenzymes (Lee et al., 2007) and probably is used in the central nervous system (CNS) as a neurotransmitter or neuromodulator (Zulfiqar and Husein, 2004).

b) As zinc is a constituent of metalloenzymes so, it is involved in a large number of biological processes related to child motor development.

c) Zinc is essential for the metabolism of vitamin A.

d) Zinc is involved in the synthesis of DNA and RNA without which cells cannot reproduce themselves and growth and development would be impossible.

e) Zinc is needed for cell replication and is thus essential for regeneration of the intestinal mucosa.

f) Zinc is essential for the healing of wounds and the turnover of epithelial cells necessary to maintain healthy skin.

Zinc is found in a wide range of foods; the richest sources are animal protein (meat, eggs and dairy products) shellfish, pulses, nuts and whole meal grains. White flour is a poor source of zinc because the zinc is mainly found in the outer layers of the grain and because the fiber in grain contains phytates, which inhibits the absorption of minerals. Zinc plays an important role in the promotion of normal growth and development and is an element in the enzymes that work with red blood cells, which move $\mathrm{CO}_{2}$ from tissues to lungs. It also helps to maintain an effective immune system. Zinc deficiency in malnourished children contributes to growth failure and susceptibility to infections. It is also associated with complications of child birth. This deficiency usually occurs where malnutrition is prevalent (UNICEF, 2001). Medium-to-severe zinc deficiency disturbs several biological functions such as gene expression, protein synthesis, immunity, skeletal growth and maturation, gonad development and pregnancy outcomes, including taste perception and appetite (Onyezili et al., 1993). According to Penny severe zinc deficiency is responsible for dwarfism and failure to mature in young Iranian and Egyptian youths eating a diet consisting largely of whole meal bread. Penny observed that lesser degrees of zinc deficiency are more common now than was appreciated and the sub clinical deficiency of zinc contributes to an increased incidence and severity of common but important infections such as diarrhea and pneumonia. Diarrhea is associated with an increased loss of zinc in faces. (Onyezili et al., 1993) also stated that zinc deficiency increases the risk of mortality from diarrhea, pneumonia and malaria by 13 to 21 percent and unlike other micronutrients of public health importance has often subtle rather than dramatic clinical features.

Despite the widespread presence of zinc in common foods, dietary deficiency is common because bioavailability of zinc is reduced by the co-existence of inhibitory substances such as fiber and phytate in foods of vegetable origin. These substances inhibit the absorption of zinc. The diets of the poorest populations often lack zinc being dependent on vegetables staples with little assess to animal products especially flesh products such as meat, fish and offal which are the richest sources of easily assimilated zinc. Children in poor communities do not manifest obvious signs of zinc deficiency but have often been found to have low plasma zinc levels compatible with sub-clinical zinc deficiency. Zinc supplements helped blunt the most severe malaria 
cases in children. The deficiency of Zinc can be prevented by intake of zinc supplement and foods rich in protein.

\section{Child health and development}

Evidence suggests that micronutrient deficiencies may be associated with problems in early development and behavior. Iron deficiency is the most common nutritional deficiency in the world and a major cause of anemia particularly during infancy and toddlerhood when there is rapid growth and a high nutritional demand (Blair, 2006). Less is known about the prevalence of zinc deficiency, but supplementation trials in developing countries suggest low zinc intake among infants and toddlers (Takeda, 2001), which leads to the consensus that both iron and zinc deficiency are major public health problems (Stoltzfus, 2001; Hotz, 2001). In low-income societies (like Bangladesh), where dietary resources are inadequate to meet children's requirements for iron and zinc, micronutrient supplementation or fortification may be necessary (UNICEF, 2011).To identify the dietary information related to child's cognitive, health and development process-A cognitive processing model has been demonstrated (Black, 2004).

A cognitive-processing model has been demonstrated to be useful in understanding self-reports of other behaviors. Figure 1 graphically presents a simple representation of cognitive processing about food intake and questioning about that intake (Shaffer, 1985). This model was adapted from Shaffer and has three structural elements: a sensory register, short-term memory (STM), and long-term memory (LTM). The sensory register is a boundary structure that screens the information to which children attend. When information in the environment is perceived, it is used in STM, also known as working memory. All conscious intellectual activity is believed to occur in STM, however, most people can hold few items of information in STM (perhaps about five) at any one time. Some portion of the information in STM is transferred to storage for later

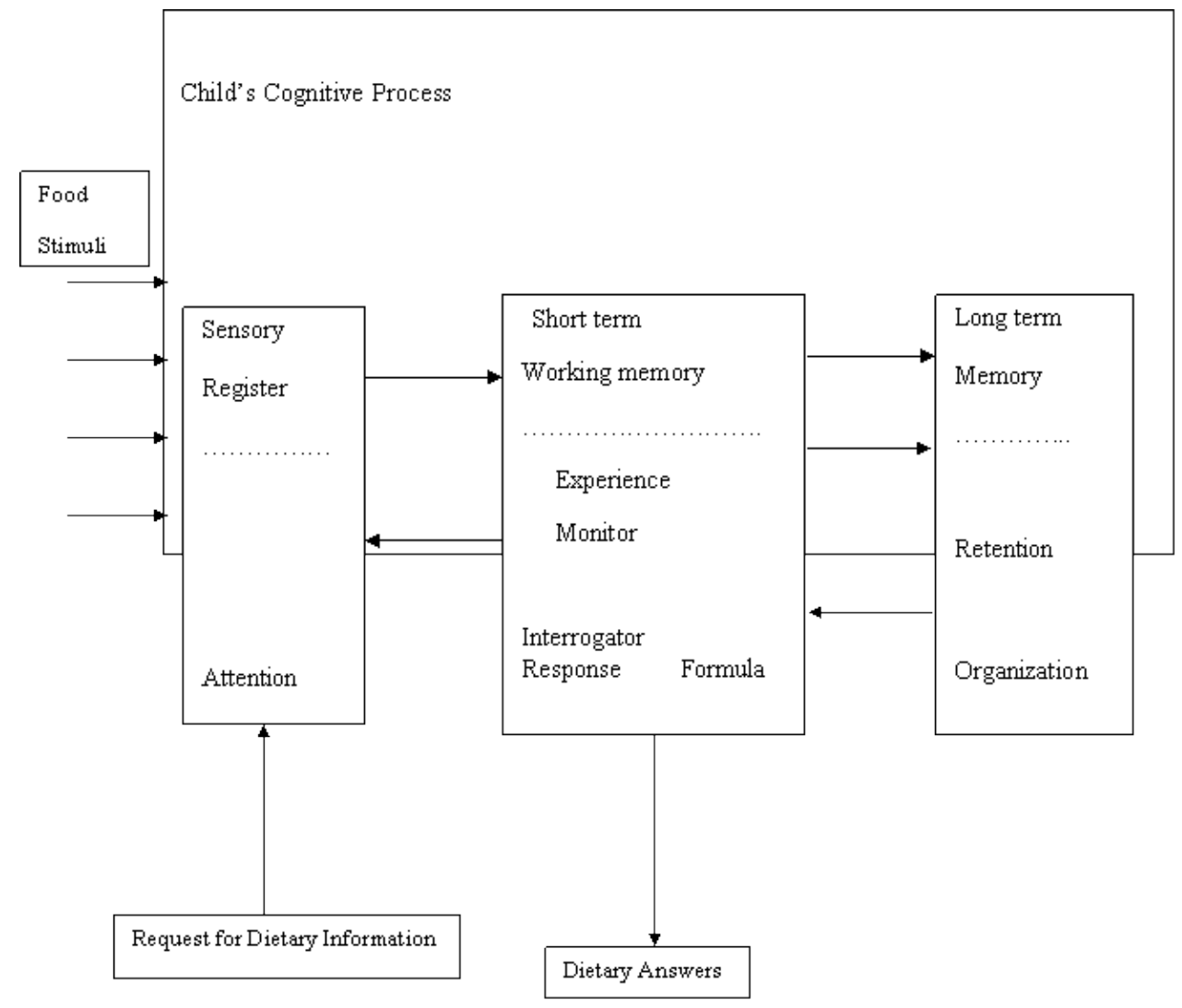

Figure 1. A model of a child's cognitive processing of food information.

\section{Conclusions}

All efforts should be made that children take a well balanced nutritious food by encouraging them to consume green leafy vegetables, soy beans, seasonal fruits, milk and dairy products, fish, eggs, chicken and other food stuff. However, the prevailing dogma in nutritional science that a balanced diet is sufficient to meet all the nutritional requirements has been challenged (Singh M, 2004). According to the recommendations of United 
Nations Sub-committee on Nutrition, it is not possible to meet the requirements of 100 percent recommended dietary allowance (RDA) of micronutrients from dietary sources alone (Jill et al., 2001). Nutritional supplements are thus mandatory to improve physical growth and mental development and prevent occurrence of common day-today infections. Healthy children do provide a solid foundation to the society in order to ensure optimal resource development of a country.

\section{Conflict of interest}

None to declare.

\section{References}

Algarín C, P Peinaro, M Garrido, F Pizzaro and B Lozoff, 2003. Iron deficiency anemia in infancy: long-lasting effects on auditory and visual system functioning. Pediatrics Res., 53: 217-223.

Allen LH, 2001. Micronutrients: ${ }^{2020}$ Focus 5 Healths and Nutrition Emerging and Reemerging Issues in Developing Countries. Brief 10 of the International Food Policy Research Institute 2001.

Bananowski T, 1988. Validity of self report of physical activity: information processing approach. Res. Q. Exerc. Sport., 59: 314-27.

Beard J, 2001. Iron biology in immune function, muscle metabolism and neuronal functioning. Journal of Nutrition, 131: 568-580.

Black MM, 2003. Micronutrient deficiencies and cognitive functioning. Journal of Nutrition, 3927-3931.

Black MM, 2004. The evidence linking zinc deficiency with children's cognitive and motor functioning. Journal of Nutrition. 1473-1476.

Blair C, 2006. How similar is fluid cognition and general intelligence? A developmental neuroscience perspective on fluid cognition as an aspect of human cognitive ability. Behav. Brain Sci., 29: 109-125.

Caballero B, 2002. Global patterns of child health: the role of nutrition. Ann. Nutri. Metab., 1: 3-7.

Children's Fund, World Health Organization, 2001. A Guide for New Program Managers United Nations University.

De Ungria M, R Rao, JD Wobken, M Luciana, CA Nelson and MK Georgieff, 2000. Perinatal iron deficiency decreases cytochrome c oxidase (CytOx) activity in selected regions of neonatal rat brain. Pediatrics Res., 48: 169-176.

Denis LA and VDW Denis, 2005. Spirulina as a food complement to improve health and cognitive development. Nutrition and cognitive development-Antena technologies. Retrieved from www.antenna.ch

Draper A,1996. Child development and iron deficiency. The Oxford Brief.

Frederickson CJ, 1989. Neurobiology of zinc and zinc-containing neurons. Int. Rev. Neurobiol., 31: 145-238.

Georgieff MK and SM Innis, 2005. Controversial nutrients that potentially affect preterm neurodevelopment: essential fatty acids and iron. Pediatrics Res., 57: $99-103$.

Hotz $\mathrm{C}$ and $\mathrm{KH}$ Brown, 2001. Identifying populations at risk of zinc deficiency: the use of supplementation trials. Nutr. Rev., 59: 80-4.

Indian Pediatrics, 2004. The role of zinc in child health in developing countries: Taking the science where it matters, Editorial 41: 429-433.

Jill SH, M Jeffrey, Kaczorowski, CA Andrew, A Peggy and GS Peter, 2001. Iron deficiency and cognitive achievement among school-aged children and adolescents in the United States. Pediatrics, 107: 1381-1386.

Arnold LE and AD Robert, 2005. Zinc in attention-deficit/hyperactivity disorder. Journal of Child and Adolescent Psychopharmacology Mary Ann Liebert Inc., 15: 619-627.

Lee RM, W Nancy, M Rachel and F Tara, 2007. Vitamins and minerals functioning as antioxidants with supplementation considerations. University of Florida. Retrieved from http://dairy.ifas.ufl.edu/rns/2007/McDowell.pdf

Lozoff B, E Jimenez, J Hagen, E Mollen and A Wolf, 2000. Poorer behavioral and developmental outcome more than 10 years after treatment for iron deficiency in infancy. Pediatrics, 105 (4).

Lozoff B, 2000. Perinatal iron deficiency and the developing brain. Pediatrics Res., 48: 137-138.

Maureen MB, 1998. Zinc deficiency and child development. American Journal of Clinical Nutrition, 68: 464-9.

Maureen MB, 2003. The evidence linking zinc deficiency with children's cognitive and motor functioning. Journal of Nutrition, 133: 1473-1476.

Ozgur Y, A Cemal, S Ahmet, C Adnan, S Teoman and OC Ayhan, 2004. Zinc status in autistic children. The Journal of Trace Elements in Experimental Medicine, 17: 101-107.

Onyezili FN, IS Alo and A Tinorgah, 2003. Micronutrient deficiency control and child survival in Nigeria, In: Akinyele IO, Onimawo IA, Uwaegbute AC, Child Survival and the Right to Adequate Nutrition. 2003; 
November 26th - 29th. (Proceedings of the 34th Conference and Scientific Meeting of Nutrition Society of Nigeria.Held at Michael Okpara University of Agriculture, Umudike, Abia State).

Piaget J, 1997. The Language and Thought of the Child.OH: World Publishing Company (Cleveland).

Pollitt E, 1993. Iron deficiency and cognitive function. Annual Review of Nutrition, 13: 521-537.

Ruff HA and MK Rauthbart, 1996. Attention in Early Development. NY: Oxford University Press (New York).

Sally GM and A Cornelius, 2001. A Review of studies on the effect of iron deficiency on cognitive development in children. Journal of Nutrition, 131: 649-668.

Shaffer DR, 1985. Developmental psychology, theory, research and application. Monterey, CA: Brooks/Cole Publishing 1985.

Shinjini B and T Sunita, 2001. Zinc and cognitive development. British Journal of Nutrition, 85: 139-145.

Singh M, 2004. Role of micronutrients for physical growth and mental development. Indian Journal of Pediatrics, 71: 59-62.

Gregor SMG and CC Ani, 1999. The role of micronutrients in psychomotor and cognitive development. British Medical Bulletin, 55: 511-527.

Stoecker BJ, Y Abebe, LT Hubbs, TS Kennedy, RS Gibson, I Arbide, A Teshome, J Westcott, NF Krebs and KM Hambidge, 2009. Zinc status and cognitive function of pregnant women in Southern Ethiopia. European Journal of Clinical Nutrition, 1-3.

Stoltzfus RJ, 2001. Defining iron-deficiency anemia in public health terms: a time for reflection. Journal of Nutrition, 131:565-567.

Takeda A, 2001. Zinc homeostasis and functions of zinc in the brain. Biometals, 14: 343-351.

Tom B and BD Suzanne, 1994. A cognitive model of children's reporting of food intake. American Journal of Clinical Nutrition, 59: 212-7.

Tsunenobu T, LG Robert, LR Sharon, GN Kathleen and RC Victoria, 2003. Effect of zinc supplementation of pregnant women on the mental and psychomotor development of their children at $5 \mathrm{y}$ of age. American Journal of Clinical Nutrition, 77: 1512-6.

United Nations Children Fund; United Nations University; World Health Organization 2011. Iron Deficiency Anaemia Assessment, Prevention and Control: A guide for programme managers; WHO/NHD/01.3.

Zulfiqar AB and LD Husein, 2004. The role of zinc in child health in developing countries: taking the science where it matters. Indian Pediatrics, Editorial 41: 429-433. 